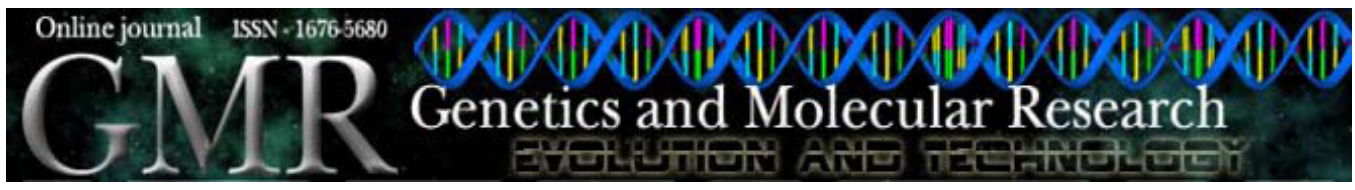

\title{
Genetic variation of Casuarina equisetifolia subsp equisetifolia and $C$. equisetifolia subsp incana populations on the northern coast of Senegal
}

\author{
A.L. Ndoye ${ }^{1}$, O. Sadio ${ }^{2}$ and D. Diouf ${ }^{1}$ \\ 'Département de Biologie Végétale, Laboratoire de Biotechnologies Végétales, \\ Faculté des Sciences et Techniques, Université Cheikh Anta Diop, \\ Dakar-Fann, Dakar, Sénégal \\ ${ }^{2}$ UMR 195 LEMAR, IRD, Centre de Bel Air, Dakar, Sénégal \\ Corresponding author: D. Diouf \\ E-mail: diaga.diouf@ucad.edu.sn
}

Genet. Mol. Res. 10 (1): 36-46 (2011)

Received December 7, 2010

Accepted December 23, 2010

Published January 11, 2011

DOI 10.4238/vol10-1gmr986

\begin{abstract}
The genetic variation of 70 individual samples of $\mathrm{Ca}$ suarina equisetifolia (L. Johnson) subsp equisetifolia and C. equisetifolia subsp incana growing along the northern coast of Senegal was analyzed with RAPD markers. Of the 160 primers tested, five were chosen; they generated 1396 reproducible bands and 61 polymorphic bands that were scored. This result showed a narrow genetic variation among (4.36\%) and within (5.90\%) C. equisetifolia subsp equisetifolia and $C$. equisetifolia subsp incana plantation sites. The genetic variation at each site revealed a high degree of polymorphism in Potou $(5.90 \%)$ and low diversity in Retba (3.06\%). In the dendrogram analyses, each sampling site was formed by two main groups. Similar results were found for the dendrograms based on the RAPD data gathered from the five different sites. These dendrograms revealed several polytomies in one of the subgroups, suggesting replication of the same specimens in different sites along the Senegalese coast. The RAPD data support the hypothesis that these populations are of the same provenance, subject to
\end{abstract}


hybridization and inbreeding depression.

Key words: Casuarina equisetifolia subsp equisetifolia; RAPD; C. equisetifolia subsp incana; Genetic diversity; Molecular marker; Northern coast of Senegal

\section{INTRODUCTION}

Casuarina equisetifolia (L. Johnson) $2 \mathrm{n}=18$ is a nitrogen-fixing tree including two subspecies, C. equisetifolia subsp incana (6-12 m) and C. equisetifolia subsp equisetifolia (7-35 m) (Midgley et al., 1983; Wilson and Johnson, 1989). They are distributed in Australia, Southern Asia, Pacific Islands, tropical and subtropical regions, where native or introduced populations are grown but the subspecies equisetifolia is more common outside its area of origin (Wilson and Johnson, 1989; Diouf et al., 2008). In these regions, C. equisetifolia is used for landscaping, timber, medicine, dye, pulp, tannin, wood fuel production, soil stabilization, reforestation of marginal ecosystems, amenity planting, and land fertilization (Pan et al., 1996). Because it is salt and drought tolerant, and fast growing, C. equisetifolia is also used as windbreaks and sand-shifting control in many parts of the world such as on the northern coast of Senegal (9700 ha) and Southern China (30,000 ha) (Mailly et al., 1994; Zhong et al., 2005). Due to its importance, there is a major effort underway focused on selecting and planting elite clones of C. equisetifolia in Australia and India, but the efficiency of this approach requires the genetic characterization of available genetic resources (Yasodha et al., 2004).

In recent years, intensive studies have been done on the genetic diversity of $C$. equisetifolia regarding the socio-economic and ecological importance of this tree. For this purpose WARD's technique based on the hierarchical methods of grouping was used in 505 phenotypically superior trees of $C$. equisetifolia. This study showed genetic variability between C. equisetifolia trees in different plantations and suggested hybridization among clones (Kumar and Gurumurthi, 2000). The recent inflow in applying molecular techniques in Casuarina chloroplast or nuclear genomes has provided a better understanding of species and subspecies delineation. The comparison of matk sequences, a fast evolving chloroplast gene, showed that C. equisetifolia from the Philippines and Malaysia are very close but genetically distant to the Australian provenances (Steane et al., 2003). Other DNA markers such as ISSR (inter-simple sequence repeat), fluorescent-ISSR or combined with morphological traits were widely used for assessing the genetic diversity of $C$. equisetifolia. They showed polymorphism between clones of C. equisetifolia from Australia and suggested that morphological markers can be used in combination with molecular markers in genetic variability assessment and breeding programs (Yasodha et al., 2004; Kamalakannan et al., 2006). Genetic variation at the population level was also studied in a $C$. equisetifolia plantation in Taiwan by using random amplified polymorphic DNA (RAPD) markers. The data support a wide genetic variation in native $C$. equisetifolia and introgressive hybridization among C. equisetifolia, C. glauca and C. cunninghamiana (Ho et al., 2002a,b). RAPD is a simple method based on DNA amplification developed by Williams et al. (1990). This technique does not require high quality or large amounts of DNA. It is useful for genetic diversity assessment at different levels such as population, species and genus but also for identifying interspecific and introgressive hybridization (Ho et al., 2002b).

In this study, our objective was to assess genetic diversity of $C$. equisetifolia popula- 
tions from 5 different sites located on the northern coast of Senegal and to investigate their genetic relationship.

\section{MATERIAL AND METHODS}

\section{Plant materials}

Branchlets of C. equisetifolia were collected from trees growing in 5 different sites (Guédiawaye, Malika, Notto, Potou, and Retba) on the northern Senegalese coast. In each site, 15 trees including 5 female, 5 male and 5 hermaphrodite plants identified by GPS were chosen for branchlet collection (Table 1). The two subspecies equisetifolia and incana were identified by observing the branchlets and cones with binoculars (SZ-PT, Olympus, Japan). A few specimens were removed from the analysis because they did not amplify. The selection of the tree was based on three morphological criteria: circumference, height and the trunk. The branchlet samples were stored in plastic bags and dried with silica gel before DNA extraction.

\section{DNA isolation, amplification and electrophoresis}

DNA of C. equisetifolia was extracted according to the protocol developed by Badiane et al. (2004). After extraction, DNA was dissolved in 0.1X TE, quantified with a spectrophotometer (8500-II Spectrophotometer, Techcomp, Ltd., Hong Kong) and stored at $-20^{\circ} \mathrm{C}$. Amplification reactions, gel electrophoresis and staining were performed according to the protocol described by Badiane et al. (2004). Each reaction was repeated three times to ensure reproducibility of the results.

\section{Scoring, RAPD data and statistical analyses}

Bands were scored on the basis of presence (1) or absence (0) across the samples. Bands present across all the samples were excluded from the analysis because they are not informative. Data matrices generated by RAPD were submitted to principal component analysis and analysis of molecular variance (AMOVA).

\section{Multivariate analysis}

A normalized principal component analysis using the statistical package ADE-4 coupled with a hierarchical cluster analysis was performed for grouping the samples by their similar characters and the "starters were considered as variables". The C. equisetifolia samples were projected as individuals on a factorial plane including the first two axes. An ascending hierarchical clustering of the individuals was carried out by using the coordinates of the individuals on the factorial axes as similarity matrix, the Euclidean distance and the Ward method. The treatments were carried out with the R software (version R-2.9.0) using the ADE4 package for generating a dendrogram. The similarities shown on the dendrogram ranged from zero (high similarity) to 25 (lower similarity).

\section{RESULTS}




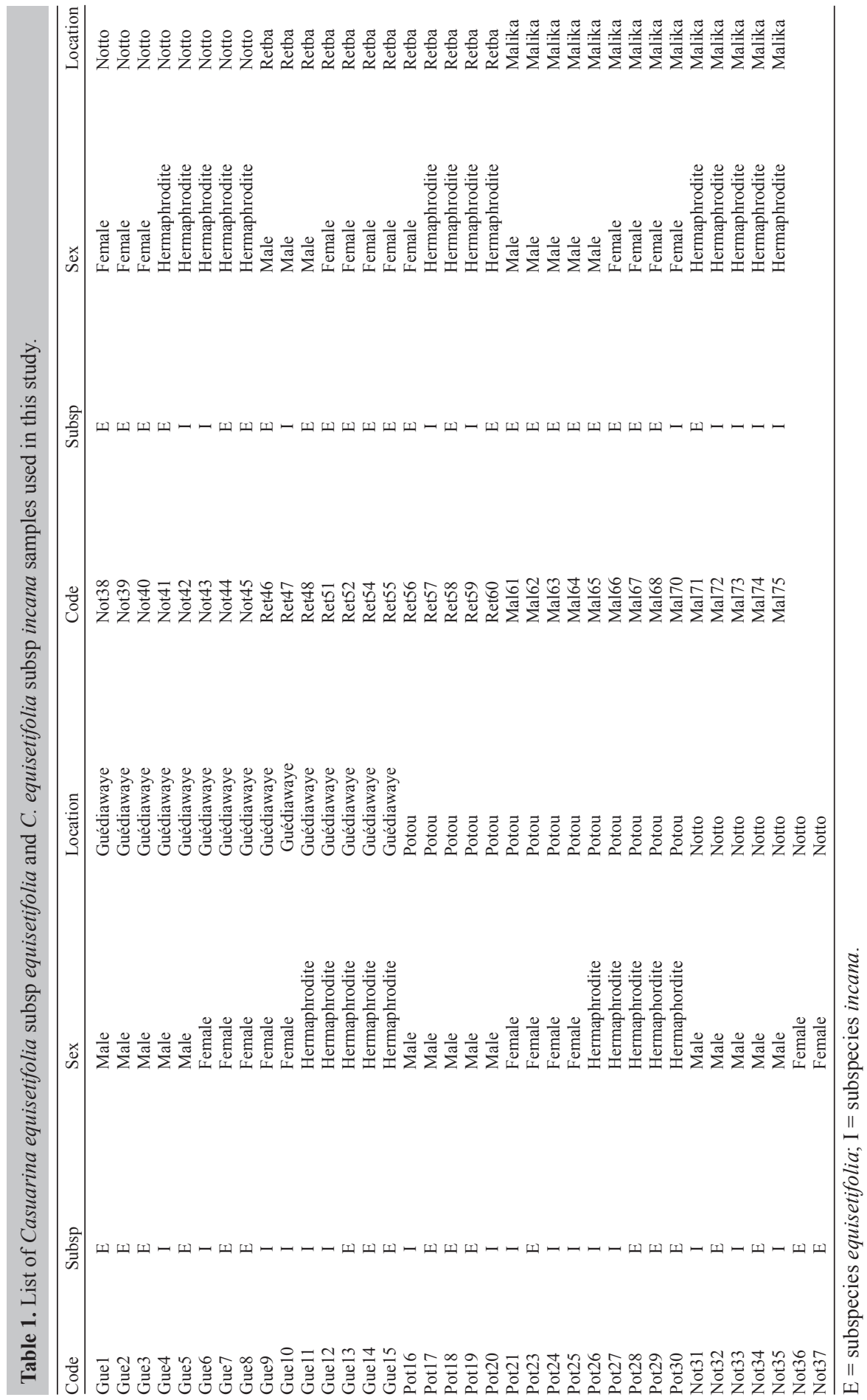




\section{RAPD polymorphism}

Among the 160 primers (Kit OPA, OPB, OPF, OPN, OPO, OPQ, OPR, and OPT; Génosphère Biotechnologies, Paris, France) tested, only 5 were chosen in this study (Table 2). Other primers were removed from the analysis because they did not show any polymorphic bands or due to the complexity of their amplification. The primers OPO15 and OPT1 did not show any polymorphic bands in the populations from Retba and Malika, respectively, compared to the OPF4 and OPT16, which generated the highest percentage of polymorphic bands in each site (data not shown). The genetic variation assessed in each site revealed a high polymorphism in Potou (5.90\%) and a low diversity in Retba (3.06\%) (data not shown). In total, 1396 reproducible bands were amplified, of which 61 were polymorphic (4.36\%). The average number of total bands and the number of polymorphic bands per primer were $279.2 \pm 65.404$ and $12.2 \pm 4.5$, respectively. The primer OPF4 showed the highest percentage of polymorphic bands (7.01\%).

Table 2. Nucleotide sequences and name, total number of bands, number of polymorphic bands, and percentage
of polymorphic bands for each primer.
\begin{tabular}{lcccc}
\hline Name and sequence of primers & Total No. of bands & No. of polymorphic bands & Percentage of polymorphic bands \\
\hline OPA7 & 5'GAAACGGGTG3' & 308 & 9 & 2.92 \\
OPF4 & 5'GGTGATCAGG3' & 228 & 16 & 7.01 \\
OPO15 & 5'TGGCGTCCTT3' & 192 & 6 & 3.125 \\
OPT1 & 5'GGGCCACTCA3' & 329 & 14 & 4.25 \\
OPT16 & 5'GGTGAACGCT3' & 339 & $12.2 \pm 4.5$ & 4.71 \\
Average & $279.2 \pm 65.404$ & 61 & 4.36 \\
Total & 1396 &
\end{tabular}

Genetic variation within sites

The samples from Guédiawaye were clustering in two main groups in the dendrogram (Figure 1A). The first group was formed by two main subgroups. Subgroup 1 includes only the subspecies equisetifolia (the males Gue5, Gue1, and the female Gue7), but the female Gue8 and the male Gue3 were isolated from the rest of the subgroup forming a polytomy. The second subgroup encompasses small subgroups such as the incana specimens (hermaphrodite Gue12 and the female Gue9), which are distant from the equisetifolia hermaphrodite Gue13. The equisetifolia hermaphrodite Gue14 and the incana female Gue10 were clustered together. In contrast, the subspecies equisetifolia (hermaphrodite Gue15 and the male Gue2) are located at the base of this subgroup. The second group includes only the subspecies incana (the female Gue6 and the male Gue4), but the incana hermaphrodite Gue11 showed a high coefficient of dissimilarity with the other specimens.

The dendrogram carried out for the population from Potou showed two groups (Figure 1B). The incana female Pot 25 and the equisetifolia hermaphrodite Pot30, isolated at the base of the first group, seem to have a high coefficient of dissimilarity with the rest of the group. In contrast, the second group showed that the equisetifolia female Pot23 and the equisetifolia hermaphrodite Pot 27 were very similar to each other, but had a strong coefficient of dissimilarity with the other individuals from the same group. The incana female Pot21 and the equisetifolia hermaphrodite Pot29 were forming a polytomy. In the same group, the equisetifolia hermaphrodite Pot 28 was isolated from the others.

The population planted in Notto can be divided into two groups; one of them in- 

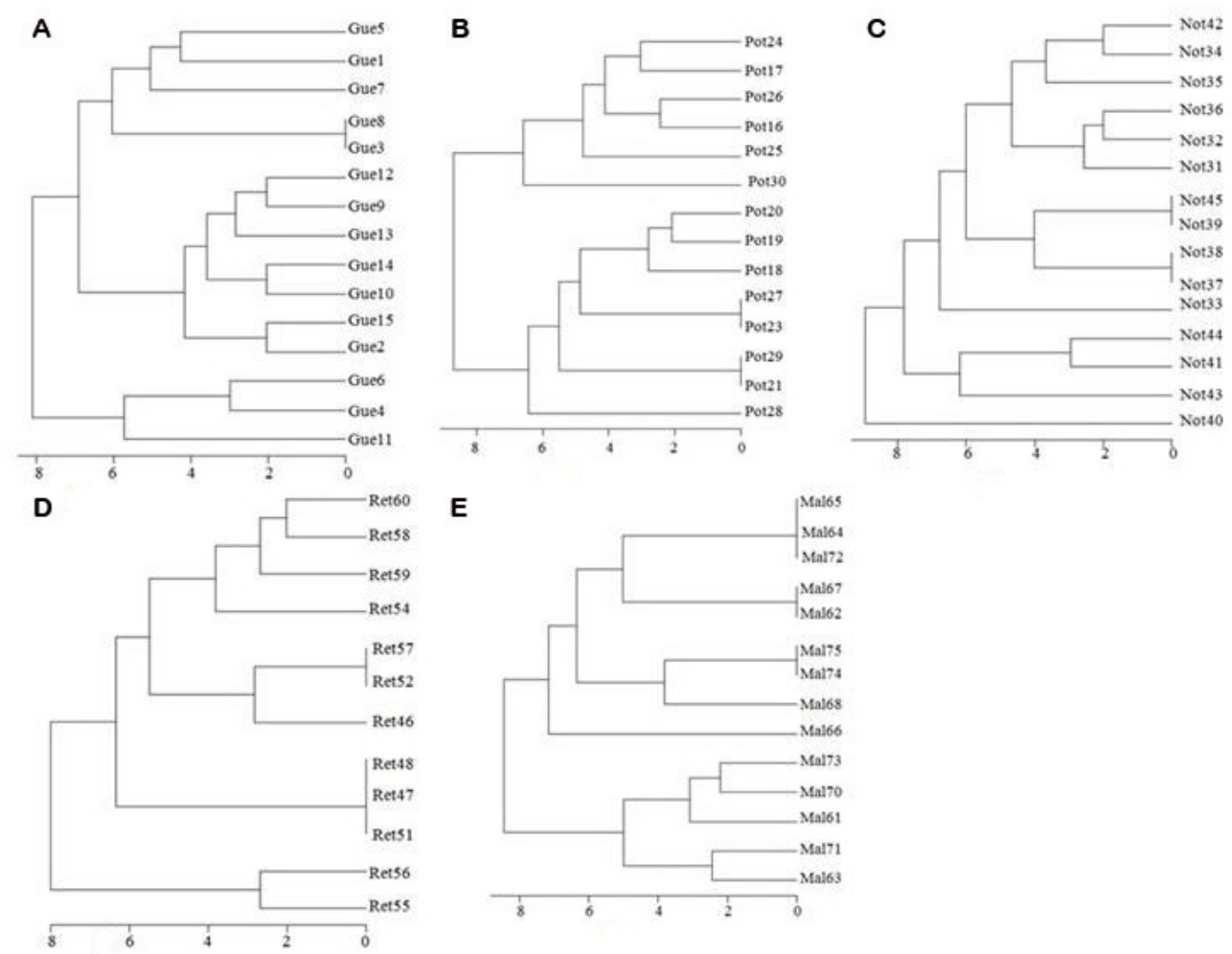

Figure 1. Dendrogram showing the similarity among Casuarina equisetifolia subsp equisetifolia and C. equisetifolia subsp incana populations based on RAPD data. A. Site of Guédiawaye. B. Site of Potou. C. Site of Notto. D. Site of Retba. E. Site of Malika.

cludes only the equisetifolia female Not40, which was isolated in the dendrogram (Figure 1C). The second group included several subgroups such as the incana hermaphodite Not 42 and the equisetifolia male Not34, which showed a higher coefficient of dissimilarity with the equisetifolia male Not35. The equisetifolia (male Not32 and the female Not36) are clustering together and the incana male Not31 is located at the base of this subgroup. However, the equisetifolia (the female Not39 and the hermaphrodite Not45, and the females Not37 and Not38) also formed a polytomy. But the incana male Not33 was isolated from the rest of the subgroup. The equisetifolia hermaphrodites Not41, Not43 and incana hermaphrodite Not44 formed the last subgroup.

The population from Retba formed two groups (Figure 1D). The equisetifolia female Ret52 and the hermaphrodite incana Ret57 clustered together to form a polytomy in our study. A second polytomy was observed between the incana male Ret47 and the equisetifolia specimen (male Ret48 and the hermaphrodite Ret51). These three samples showed a high coefficient of dissimilarity with the other specimens in this group. The equisetifolia (the female Ret55 and the hermaphrodite Ret56) constitute a group that is very dissimilar from the first group.

Two main groups were described for the Casuarina growing in Malika (Figure 1E). 
The equisetifolia males (Mal64 and Mal65) and the incana hermaphrodite Mal72 formed a subgroup at the top of the dendrogram. This subgroup also includes the equisetifolia (the male Mal62 and the female Mal67) specimens. The second subgroup encompasses the incana hermaphrodites Mal74 and Mal75 and the equisetifolia female Mal68. The third subgroup includes only the equisetifolia female Mal66. In the second group, the incana (the female Mal70 and the hermaphrodite Mal73) are more similar than with the equisetifolia male Mal 61. The equisetifolia male Mal63 and the hermaphrodite Mal71 are clustering at the base of this subgroup.

\section{Genetic variation between sites}

The genetic relationship of Casuarina grown at the five sites (Guédiawaye, Malika, Notto, Potou, and Retba) is shown on the dendrogram of Figure 2 where two main groups are identified. The first group includes $80 \%$ of Casuarina from Guédiawaye, $64.36 \%$ from Potou, $35.7 \%$ from Malika, $33.33 \%$ from Notto, and $16.66 \%$ from Retba. This group also includes four subgroups, 21.43 and $25.71 \%$ of the collected specimens belonging to the subspecies incana and equisetifolia, respectively. The first subgroup did not have any specimens from Malika and Retba but showed similarity between Casuarina grown in Guédiawaye, Notto and Potou. The C. equisetofolia subsp incana (Not35, Gue9 and Pot26) were clustering at the top of the dendrogram. While in the second subgroup only two specimens from Guédiawaye (Gue12) and Malika (Mal66) were similar, the third and the fourth subgroups did not have any similar specimens. In the fourth subgroup, the incana (Gue11, Pot16, Pot25, Not33) are clustering together. The second group includes four subgroups, a high number of polytomies, with 14.23 and $38.57 \%$ of the collected specimens belonging to the subspecies incana and equisetifolia, respectively. In this second group, some equisetifolia subspecies were clustering among several subgroups. Pot18 and Mal61 formed a polytomy. The other equisetifolia subspecies clustered in separate subgroups like Ret58 and Ret60, Not34 and Ret46, Not37 and Not38, Mal67, Mal62, Ret56, Not36, Gue2, and Pot19.

\section{DISCUSSION}

To our knowledge, this is the first time that attention has been paid to the genetic diversity of Casuarina populations growing on the northern coast of Senegal, which were planted a decade ago. Unfortunately, no records have been reported concerning the origin of the specimens introduced in these areas. For this purpose it is important to understand the genetic relationship of C. equisetifolia growing within and among these sites before implementing a breeding program.

\section{Genetic variation}

Our data showed a low percentage of polymorphic bands (4.36\%) among the amplified fragments. These results are in agreement with the conclusions of Ho et al. (2002b) who found low genetic diversity in a $C$. equisetifolia population growing in Taiwan by using RAPD. Similar results based on ISSR markers were reported between Allocasuarina littoralis provenances by Kamalakannan et al. (2006). In contrast, the chloroplastic gene matk was not efficient in differentiating C. equisetifolia provenances (Steane et al., 2003).

The studies carried out on international provenances showed high genetic diversity 


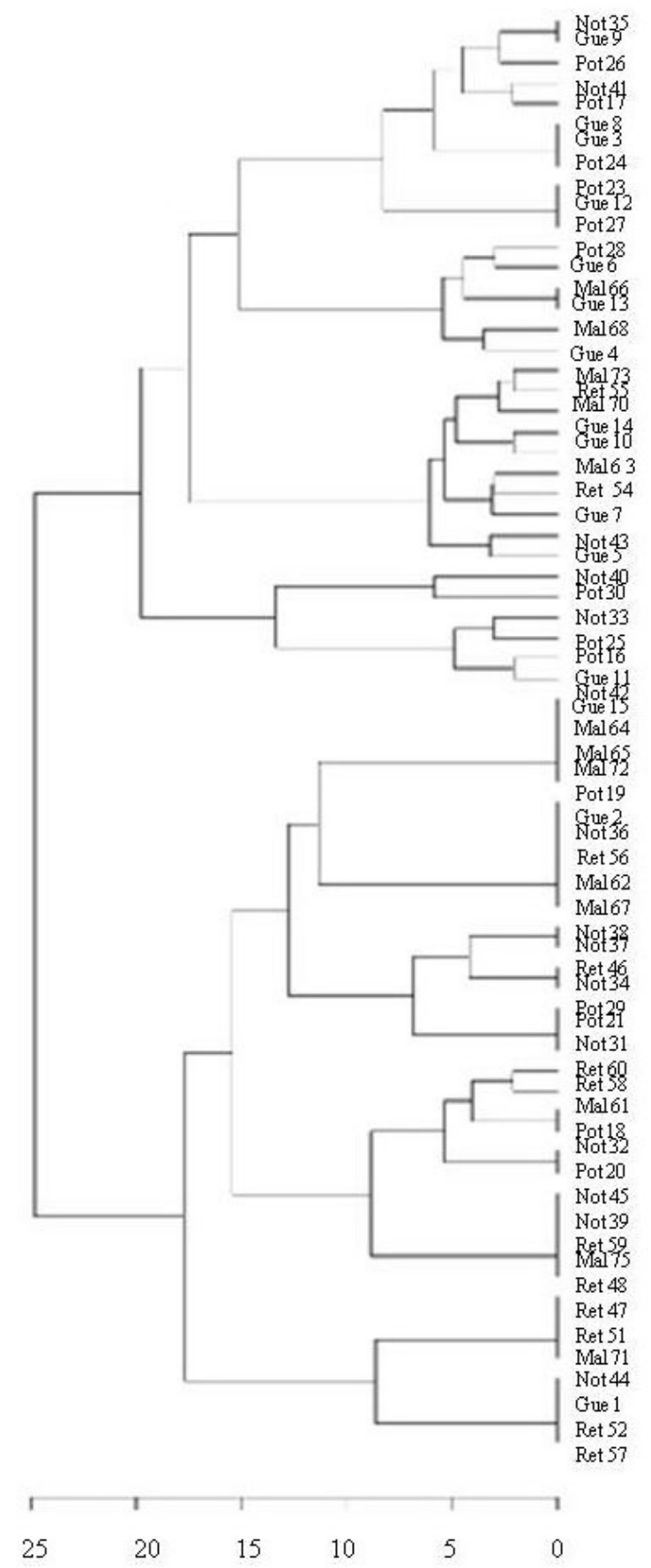

Figure 2. Dendrogram showing the dissimilarity among Casuarina equisetifolia subsp equisetifolia and C. equisetifolia subsp incana populations growing in five different sites in the Northern coast of Senegal, based on RAPD data. 
among the C. equisetifolia (Ho et al., 2002a). Obviously, international provenances are geographically isolated and this phenomenon limits gene flow between provenances and is susceptible to inducing genetic variation due to adaptation to the environmental conditions, which supports the hypothesis of bottleneck effects. In our study, this low genetic variation suggests that the specimens grown on the northern coast of Senegal might have the same origin or could be the result of hybridization due to the cross-pollination occurring in this species. This conclusion supports the hypothesis that the plantation in this area was probably created in an effort to solve an immediate environmental problem such as windbreaks and sand-shifting control than for genetic variation. High genetic variation in $C$. equisetifolia provenances was reported by several studies by using morphological and molecular markers alone or in combination (Pinyopusarerk and Williams, 2000; Yasodha et al., 2004; Kamalakannan et al., 2006). Studies based on amplified fragment length polymorphism (AFLP) markers revealed a high genetic variation among provenances of $C$. equisetifolia (Huang et al., 2008) and other tropical trees such as Moringa oleifera (Muluvi et al., 1999) and Tectona grandis (Shrestha et al., 2005). This high variation could be attributed to the high number of fragments generated by AFLP, which increases the chance of finding polymorphic bands.

\section{Relationship among accessions}

Dendrogram analyses showed two main groups and at least one polytomy for each site but in Notto several subgroups were indentified, one of them includes only one accession (Not40) (Figure 1A-E). Similar results were described in Figure 2, which represented the samples gathered from all the sites. Interestingly, the clustering of the specimens in the dendrograms and those forming polytomies was not linked to the site and the sex of the plants. This finding suggested putative hybridization between the parents of some specimens planted in different locations along the Senegalese coastline. Hybridization was also described as occurring between C. equisetifolia, C. glauca and C. cunninghamiana (Ho et al., 2002b; Gaskin et al., 2009). These hybrids could be very problematic for biological control because they can be more susceptible to several diseases (virus, insects, etc.). The close relationship between the specimens would induce inbreeding depression. Inbreeding depression is known for causing embryo abortion, negatively affecting seed production, nursery performance, survival, and growth vigor in early stages in outcrossing of tropical and temperate trees (Sorensen and Cress, 1994; Williams and Savolainen, 1996; Stacy, 2001; Woods et al., 2002). This conclusion is in agreement with findings suggesting that high inbreeding depression brings about the decline of C. equisetifolia plantations (Chen and Li, 2002). It is likely that the lack of regeneration of the Casuarina population on the coastline of Senegal is the result of the combination of both inbreeding depression and the significant litter released by these trees. This litter forms a thick layer on the ground creating unfavorable conditions for seed germination. The ineffectiveness for discerning genetic relationships could be linked to RAPD technology. Sometimes RAPD amplifies different regions to the same size (co-migrating bands) in the genome that are not homologous. These bands do not share hundred percent sequence homology and represent homoplasious characters that obliviously impact negatively the analyses, leading to a lack of discrimination (Diouf and Hilu, 2005).

Our studies point to a narrow genetic variation among $C$. equisetifolia subspecies incana and C. equisetifolia subspecies equisetifolia populations growing on the northern coast 
of Senegal, which can induce inbreeding depression with the consequence of the lack of population regeneration observed in this area. Therefore, the performance of the species in the plantation cannot be optimized unless more attention is paid to the selection and improvement of the best genetic material. Furthermore, the introduction of provenances from different geographical regions to produce inter-provenance hybrids that possess desirable traits should be explored to introduce more genetic variability.

\section{ACKNOWLEDGMENTS}

We would like to thank IRD (Institut de Recherches pour le Développement, France) for supporting this project. We also thank Sunny Sheliese Crawley and the anonymous reviewers for their constructive comments.

\section{REFERENCES}

Badiane FA, Diouf D, Sané D, Diouf O, et al. (2004). Screening cowpea [Vigna unguiculata (L.) Walp.] varieties by inducing water deficit and RAPD analyses. Afr. J. Biotechnol. 3: 174-178.

Chen X and Li P (2002). Mating system and inbreeding retrogression of Casuarina equisetifolia plantation, an introduced species in Xiamen. Ying Yong Sheng Tai Xue Bao 13: 1377-1380.

Diouf D and Hilu KW (2005). Microsatellites and RAPD markers to study genetic relationship among cowpea breeding lines and local varieties in Senegal. Genet. Res. Crop Evol. 52: 1057-1067.

Diouf D, Sy MO, Gherbi H, Bogusz D, et al. (2008). Casuarinaceae. In: Compendium of Transgenic Crop Plants: Transgenic Forest Tree Species (Kole C and Hall TC, eds.). Vol. 9. Blackwell Publishing, Los Angeles, 279-291.

Gaskin JF, Wheeler GS, Purcell MF and Taylor GS (2009). Molecular evidence of hybridization in Florida's sheoak (Casuarina spp.) invasion. Mol. Ecol. 18: 3216-3226.

Ho KY, Ou CH, Yang JC and Hsiao JY (2002a). An assessment of DNA polymorphisms and genetic relationships of Casuarina equisetifolia using RAPD markers. Bot. Bull. Acad. Sin. 43: 93-98.

Ho KY, Yang JC and Hsiao JY (2002b). An assessment of genetic diversity and documentation of hybridization of Casuarina grown in Taiwan using RAPD markers. Int. J. Plant Sci. 163: 831-836.

Huang GH, Zhong CL, Su XH, Zhang Y, et al. (2008). Genetic variation and structure of native and introduced Casuarina equisetifolia (L. Johnson) provenances. Silvae Genet. 58: 79-85.

Kamalakannan R, Bartwal S, Chezhian P, Balasaravanan T, et al. (2006). Morphological and molecular diversity among Casuarina and Allocasuarina species. Biotechnology 5: 301-307.

Kumar A and Gurumurthi K (2000). Genetic divergence studies on clonal performance of Casuarina equisetifolia. Silvae Genet. 49: 57-60.

Mailly D, Ndiaye P, Margolis AH and Pineau M (1994). Fixation des dunes et reboisement avec le filao (Casuarina equisetifolia) dans la zone du littoral nord du Sénégal. For. Chron. 70: 282-290.

Midgley SJ, Turnbull JW and Johnston RD (1983). Casuarina Ecology, Management and Utilization. In: Proceedings of an International Workshop. 17-21 August, CSIRO, Canberra.

Muluvi GM, Sprent JI, Soranzo N, Provan J, et al. (1999). Amplified fragment length polymorphism (AFLP) analysis of genetic variation in Moringa oleifera Lam. Mol. Ecol. 8: 463-470.

Pan Y, Li Y and Tan TY (1996). Casuarina provenance test. For. Res. 9: 138-145.

Pinyopusarerk K and Williams ER (2000). Range-wide provenance variation in growth and morphological characteristics of Casuarina equisetifolia grown in Northern Australia. For. Ecol. Manag. 134: 219-232.

Shrestha MK, Volkaert H and Van Der Straeten D (2005). Assessment of genetic diversity in Tectona grandis using amplified fragment length polymorphism markers. Can. J. For. Res. 35: 1017-1022.

Sorensen FC and Cress DW (1994). Effects of sib mating on cone and seed traits in coastal Douglas fir. Silvae Genet. 43: 338-345.

Stacy EA (2001). Cross-fertility in two tropical tree species: evidence of inbreeding depression within populations and genetic divergence among populations. Am. J. Bot. 88: 1041-1051.

Steane DA, Wilson KL and Hill RS (2003). Using matK sequence data to unravel the phylogeny of Casuarinaceae. Mol. Phylogenet. Evol. 28: 47-59.

Williams C and Savolainen O (1996). Inbreeding depression in conifers: implications for breeding strategy. For. Sci. 42 :

Genetics and Molecular Research 10 (1): 36-46 (2011)

CFUNPEC-RP www.funpecrp.com.br 
102-117.

Williams JG, Kubelik AR, Livak KJ, Rafalski JA, et al. (1990). DNA polymorphisms amplified by arbitrary primers are useful as genetic markers. Nucleic Acids Res. 18: 6531-6535.

Wilson KL and Johnson LAS (1989). Casuarinaceae. In: Flora of Australia 3 Australian Government Publishing Service, Canberra, 100-203.

Woods JH, Wang T and Aitken SN (2002). Effects of inbreeding on coastal Douglas-fir: nursery performance. Silvae Genet. 51: 163-170.

Yasodha R, Kathirvel M, Sumathi R, Gurumurthi K, et al. (2004). Genetic analyses of casuarinas using ISSR and FISSR markers. Genetica 122: 161-172.

Zhong CL, Bai JY and Zhang Y (2005). Introduction and conservation of Casuarina trees in China. Forest Res. 18: 345-350. 